\section{AB0352 SIGNIFICANCE OF OCCULT INFECTIONS IN INFLAMMATORY ARTHRITIS PATIENTS RECEIVING BIOLOGIC THERAPIES IN EAST LONDON}

H. Alkoky, G. Qadir, O. Etomi, H. Tahir, A. Pakozdi. Rheumatology, Barts Health, London, UK

Background: Chronic hepatitis B virus (HBV) infection remains a significant global health problem. In high endemic areas like African and Asian countries, most infections occur from vertical transmission, whilst in western countries HBV is primarily acquired in adulthood. Either way, HBV can persist in infected hepatocytes lifelong, even if undetectable in the serum, allowing reactivation during immunosuppression. HBsAg carriers, those with detectable HBV viral load, or receiving concomitant corticosteroids are at greater risk. Most guidelines recommend screening for occult infections prior to starting biologic treatment including testing for HBV, HCV, HIV and TB infection.

Objectives: This study was carried out to estimate the prevalence of occult infec tions, particularly chronic HBV, in an East London rheumatology population receiving biologic therapies, and to evaluate the rate of $\mathrm{HBV}$ reactivation after starting treatment.

Methods: Inflammatory arthritis patients starting biologic therapies in Barts Health NHS Trust between August 2014 and August 2017 were identified from databases of Whipps Cross and Mile End Hospitals. Health records were reviewed focusing on $\mathrm{HBV}$ core antibody ( $\mathrm{HBcAb}$ ), HBV surface antigen ( $\mathrm{HBsAg}$ ), HBV DNA, HCV and HIV antibody status. Latent TB tests included IGRA and ELISpot assays.

Results: 757 patients were included in the study. Of those, 51 (6.7\%) were $\mathrm{HBcAb}$ positive, 6 patients $(0.8 \%)$ were HBsAg positive and two patients had low level HBV viraemia with detectable DNA antibody at baseline. $61 \%(n=31)$ of HBcAb positive patients were female, whilst $39 \%(n=20)$ were male, with median age of 58 years (IQR, 43-65). The ethnic distribution was the following: $43 \%$ asian ( $n=22$; Bangladeshi or Pakistani), $29 \%$ african or afro-Caribbean black $(n=15)$, and $18 \%$ white caucasian $(n=9)$. The underlying rheumatological conditions included rheumatoid arthritis (59\%), ankylosing spondylitis $(33 \%)$ and psoriatic arthritis $(8 \%)$. Fifteen patients $(29 \%)$ received concomitant prophylactic anti-viral therapies (lamivudine, entecavir or tenofovir). After commencing biologic therapies, no HBV reactivation was noted in the $\mathrm{HBcAb}$ positive cohort. Intermittent mild transaminitis were detected on monitoring blood tests in $22 \%(n=11)$. The rate of latent TB infection was $11.5 \%$; HCV IgG was detected in three patients, whilst HIV infection was absent in our cohort.

Conclusions: Approximately $50 \%$ of the patient population of Barts Health NHS Trust is coming from minority ethnic groups. Likely because of the diversity of the population, the prevalence of chronic HBV infection (HBsAg and HBcAb positives) in our East London rheumatology population receiving biologic therapies was higher than the national average $(0.8 \%$ vs. $0.3 \%$, respectively). No HBV reactivation was observed in the follow up period indicating that the risk of reactivation is relatively low. Nevertheless, for patients with evidence of previous infection (HBcAb positive) careful surveillance continues to be recommended.

Disclosure of Interest: None declared

DOI: 10.1136/annrheumdis-2018-eular.6451

\section{AB0353 GENETICALLY PREDISPOSITION AND PRO- INFLAMMATORY DYSREGULATIONS - CONNECTING RHEUMATOID ARTHRITIS AND MENTAL DISORDERS}

I.C. Filipescu ${ }^{1}$, L. Ghib ${ }^{1}$, P. Juan Sainz ${ }^{2}$, C. Perez-Sanchez ${ }^{3}$, C. Lopez-Pedrera ${ }^{4}$, A. Escudero ${ }^{5}$, L. Damian ${ }^{1}$, L. Muntean ${ }^{1}$, E. Collantes-Estévez ${ }^{5}$, S. Rednic ${ }^{1}$. ${ }^{1}$ Rheumatology Clinic, University of Medicine and Pharmacy, Cluj-Napoca, Romania; ${ }^{2} 3$ GENYO Centre for Genomics and Oncological Research/, University of Granada, Granada; ${ }^{3} 4$ Maimonides Institute for Research in Biomedicine of Cordoba (IMIBIC), University of Cordoba; ${ }^{4} 4$ Maimonides Institute for Research in Biomedicine of Cordoba (IMIBIC); ${ }^{5}$ Reina Sofia University Hospital/University of Cordoba, Cordoba, Spain

Background: Depressive and anxiety disorders are reported as the more prevalent psychiatric comorbidities in chronic inflammatory diseases and their occurrence has been correlated with higher serum levels of cytokines and chemokines (IL-6, IL-1, IL-12, IL-18, TNF- $\alpha$, and even TNF-RI and TNF-RII). Some single nucleotide polymorphisms (SNPs) in TNF- $\alpha$ gene have been found to play a common part in pro-inflammatory alterations in patient with rheumatoid arthritis (RA) and depressive symptoms.

Objectives: To investigate the possible influence of four single-nucleotide polymorphism in the tumour necrosis factor receptor II (TNFRII) genes and development of psychiatric disorders in patients with active RA. The second aim was to evaluate some correlation between these SNPs, the level of four chemokines and the incidence of mental disorders.

Methods: We included 89 Caucasian patients with active RA treated in a tertiary Department of Rheumatology from Cluj-Napoca. All demographic, clinical, and biological data and RA comorbidities were completed. The presence of depressions or anxiety was confirmed at the baseline by psychiatrist. Four potentially functional SNPS within TNFRSF1B (rs1061622, rs1062624, rs1061631, rs3397) were selected to be genotyped in all patients using PCR (KASPar) asASys (KBiosciences, Hoddesdon, Hertfordshire, UK) at the Centre for Genomics and Oncological Research (GENYO), Granada, Spain. Plasmatic lev els of soluble tumour necrosis factor receptor II (sTNFRII), interleukin (IL)-6, monocyte chemotactic protein (MCP) -1 and vascular endothelial growth facto (VEGF was quantified using cytofluorometry-based ELIAS technique in accordance with manufacturer's instructions using FlowCytomix kit (eBioscience, UAS) at Instituto Maimonides de Investigacion Biomedica de Cordoba - Reina Sofia Hospital, Spania.

Results: According to DAS28 (CRP) all the patient have active arthritis (5.87 \pm 0.6 ) with median disease duration of $9(4-14)$ years. Most of the patients were diagnosed with depression ( $n=33$ ), $84.8 \%$ were female. Anxiety was present at 21 subjects ( $80.9 \%$ female). In 3 cases these two mental disorders coexist.

In a subgroup with depression the polymorphism rs1061631 (GG) was significantly associated with increased level of VEGF $(p=0.007)$. A significant correlation was also found between the polymorphism rs3397 (CC/TT) and MCP-1 level $(p=0.01)$.

In a subgroup with anxiety the significant association was found between the polymorphism rs1061631 (GG) and the level of MCP-1 ( $p=0.04)$.

Conclusions: Pro-inflammatory dysregulation might be particularly relevant in some patients with RA and psychiatric illness. Amongst genetic factors that influence the susceptibility to the development of RA and psychiatric disorders some single nucleotide polymorphisms (SNPs) in TNF- $\alpha$ gene have been considered with increasing interest. Further investigation in a larger cohort is needed.

Disclosure of Interest: None declared

DOI: 10.1136/annrheumdis-2018-eular.7584

\section{AB0354 FACTORS OF THE POSITIVE OR NEGATIVE ANSWER ON THERAPY WITH DENOSUMAB IN WOMEN WITH RHEUMATOID ARTHRITIS AND OSTEOPOROSIS}

I. Dydykina, P. Kovalenko, A. Smirnov, E. Nasonov. Nasonova Reumatologic Research Institute, Moscow, Russian Federation

Objectives: To define a contribution of factors: anamnesis, clinical/laboratory markers, glucocorticoids (GC) intake, etc. on the response to therapy with denosumab in women with rheumatoid arthritis (RA) and osteoporosis (OP).

Methods: 66 postmenopausal women (mean age 59,6 $\pm 7,4$ ) with RA (mean dura-

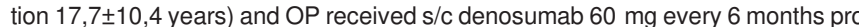
1 year. RF- positive were $72 \%$, ACCP - 74\% of patients. 34 (49\%) continued GC. At baseline and after 12 months it was carried out the dual energy $\mathrm{x}$-ray absorptiometry at 3 sites: lumbar spine (L1-L4), hip neck (HN) and distal forearm (DF) and x-ray of of hands and feet (Sharp/van der Heijde (SVH) score). After therapy it was noted the increase of BMD in L1-L4 and HN, a tendency to increase $(p=0,0529)$ in DF. Positive dynamics (increase or stabilisation of BMD) was noted in $89 \%$ patients at $\mathrm{L} 1-\mathrm{L} 4,67 \%$ - at $\mathrm{HN}$ and $60 \%$ - at DF. The erosion score was increased in $12 \%(n=8)$ patients, the joint space narrowing score $(J S N)-$ in $9 \%$ $(n=6)(p=0.0117$ and $p=0.027$, respectively). The Statistica 6.0 was used.

Results: Results of analysis of influence of statistically significant factors on the response to therapy in BMD showed that the negative response in $L 1-L 4$ is associated with GC intake ( $>3$ months in anamnesis) $(p=0,034)$ and the beginning of $\mathrm{GC}$ intake after menopause onset $(\mathrm{p}=0,023)$. In $\mathrm{HN}$ positive response is associated with higher concentration of the RF (initially and in dynamics) $(p<0,05)$ and the beginning of menopause later than RA onset $(p=0,024)$, the negative response - with $G C$ intake ( $>3$ months in anamnesis) $(p=0,024)$. In DF positive response on therapy is associated with RF-positivity $(p=0,02)$, the negative response back correlates with increase in erosion score and total SVH score: $r=-$ $0360(p<0,05)$.

In table 1 it is shown the most significant factors, which influence on SVH score dynamics (increase) after 12 months of denosumab therapy.

Abstract AB0354 - Table 1. The factors which influence on SVH score increase $(n=66)$, $\mathrm{p}<0.05$.

\begin{tabular}{ll}
\hline SVH score & \multicolumn{1}{c}{ The score increase is associated with } \\
\hline Erosion score & - lower BMD in L1-L4 (at baseline and after treatment) \\
& - higher cumulative GC dose \\
& - back correlates with BMD increase in DF \\
& - back correlates with bone alkaline phosphatase (BAP) base level \\
& - correlates with increase in JSN \\
Joint space narrowing & - presence at patients in anamnesis a surgical menopause \\
score (JSN) & - lower value of BMD dynamics (\%) in DF \\
& - correlates with increase in erosion score and total SVH score. \\
\hline
\end{tabular}

